\title{
The association between pain-induced autonomic reactivity and descending pain control is mediated by the periaqueductal grey
}

Elena Makovac ${ }^{1,3}$ (D), Alessandra Venezia ${ }^{1}$, David Hohenschurz-Schmidt ${ }^{1,2}$ (D), Ottavia Dipasquale ${ }^{1}$ (D), Jade B. Jackson $^{1,4}$ (D), Sonia Medina ${ }^{1,3}$ (D), Owen O’Daly ${ }^{1}$ (D) Steve C. R. Williams ${ }^{1}$ (D), Stephen B. McMahon ${ }^{3}$ (D) and Matthew A. Howard ${ }^{1}$ (D)

${ }^{1}$ Department of Neuroimaging, King's College London, London, UK

${ }^{2}$ Pain Research, Department Surgery \& Cancer, Faculty of Medicine, Imperial College, London, UK

${ }^{3}$ Wolfson Centre for Age Related Diseases, King's College London, London, UK

${ }^{4}$ MRC Cognition and Brain Sciences Unit, University of Cambridge, Cambridge, UK

Edited by: Harold Schultz \& Vaughan Macefield

The peer review history is available in the Supporting Information section of this article (https://doi.org/10.1113/JP282013\#support-information-section).

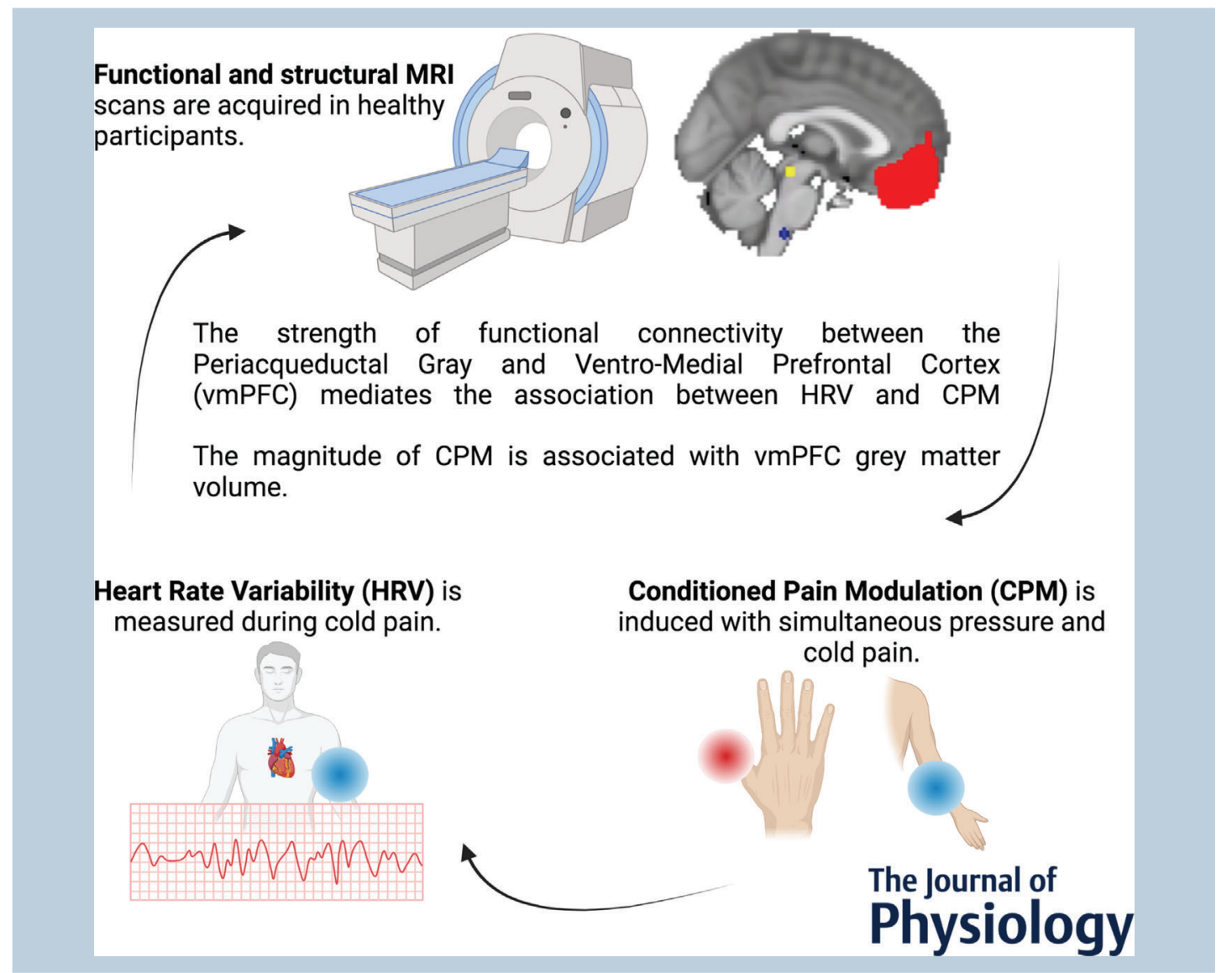

This article was first published as a preprint. Makovac E, Venezia A, Hohenschurz-Schmidt D, Dipasquale O, Jackson JB, Medina S, O’Daly O, Williams SCR, McMahon SB, Howard MA. 2020. The association between pain-induced autonomic reactivity and descending pain control is mediated by the periaqueductal grey. bioRxiv. https://doi.org/10.1101/2020.10.26.355529. 


\begin{abstract}
There is a strict interaction between the autonomic nervous system (ANS) and pain, which might involve descending pain modulatory mechanisms. The periaqueductal grey (PAG) is involved both in descending pain modulation and ANS, but its role in mediating this relationship has not yet been explored. Here, we sought to determine brain regions mediating ANS and descending pain control associations. Thirty participants underwent conditioned pain modulation (CPM) assessments, in which they rated painful pressure stimuli applied to their thumbnail, either alone or with a painful cold contralateral stimulation. Differences in pain ratings between 'pressure-only' and 'pressure + cold' stimuli provided a measure of descending pain control. In 18 of the 30 participants, structural scans and two functional MRI assessments, one pain-free and one during cold-pain were acquired. Heart rate variability (HRV) was simultaneously recorded. Normalised low-frequency HRV (LF-HRVnu) and the CPM score were negatively correlated; individuals with higher LF-HRVnu during pain reported reductions in pain during CPM. PAG-ventro-medial prefrontal cortex (vmPFC) and PAG-rostral ventromedial medulla (RVM) functional connectivity correlated negatively with the CPM. Importantly, PAG-vmPFC functional connectivity mediated the strength of the LF-HRVnu-CPM association. CPM response magnitude was also negatively correlated with vmPFC GM volume. Our multi-modal approach, using behavioural, physiological and MRI measures, provides important new evidence of interactions between ANS and descending pain mechanisms. ANS dysregulation and dysfunctional descending pain modulation are characteristics of chronic pain. We suggest that further investigation of body-brain interactions in chronic pain patients may catalyse the development of new treatments.
\end{abstract}

(Received 16 June 2021; accepted after revision 28 September 2021; first published online 13 October 2021)

Corresponding author Elena Makovac: Department of Neuroimaging, King's College London, Institute of Psychiatry, Psychology and Neuroscience, Box 89, De Crespigny Park, London SE5 8AF, UK. Email: elena.makovac@kcl.ac.uk

\begin{abstract}
The aim of this study is to investigate which brain regions mediate the association between the autonomic nervous system (ANS) and descending pain modulating mechanisms. To achieve this, healthy controls underwent a conditioned pain modulation protocol, with the aim of exploring the functioning of their descending pain modulating mechanisms. In a subgroup of these participants, we also acquired structural and functional MRI data, alongside physiological data (i.e. Heart Rate Variability -HRV- during cold pain). Overall, we observed that the CPM response was negatively associated with ventro-medial prefrontal cortex grey matter (vmPFC) and positively associated with the strength of functional connectivity between the periaqueductal grey area (PAG) and the vmPFC. Importantly, PAG-vmPFC functional connectivity mediated the strength of the association between HRV and CPM.
\end{abstract}

\title{
Key points
}

- Heart rate variability (HRV) is associated with descending pain modulation as measured by the conditioned pain modulation protocol (CPM).

- There is an association between CPM scores and the functional connectivity between the periaqueductal grey (PAG) and ventro-medial prefrontal cortex (vmPFC).

- CPM scores are also associated with vmPFC grey matter volume.

- The strength of functional connectivity between the PAG and vmPFC mediates the association between HRV and CPM.

- Our data provide new evidence of interactions between the autonomic nervous system and descending pain mechanisms.

\section{Introduction}

Nociception is modulated by descending pain control systems in the brainstem and spinal cord, under the direct influence of higher order brain areas. In humans, the efficiency of these systems can be measured using protocols such as conditioned pain modulation (CPM) (Yarnitsky, 2010). During a typical CPM trial, participants subjectively rate responses to a painful test stimulus, both on its own, and in the presence of an additional painful conditioning stimulus (Yarnitsky et al. 2015). Here, a reduction in the pain induced by the test stimulus 
when coincident with the conditioning stimulus indicates efficient descending pain control. CPM responses are, however, variable, and a reduction in pain during conditioning stimulus application is only observed in some individuals (Kemp et al. 2019). Individual differences in CPM responses may relate to factors such as age or gender (Edwards et al. 2003; Granot et al. 2008), emotions (Roy et al. 2009), personality (Nahman-Averbuch et al. 2016) or the type of protocol adopted (Kemp et al. 2019). Despite substantial interindividual variability, reports indicate CPM responses predict post-operative pain (Yarnitsky et al. 2008) and pharmacological treatment response (Yarnitsky et al. 2012), thus showing potential as a biomarker.

The bi-directional interaction between the cardiovascular system and pain is another important component of descending pain control (Bruehl \& Chung, 2004). In healthy individuals, acute pain increases sympathetic arousal and blood pressure (Kyle \& McNeil, 2014). By contrast, a reduction in pain perception is reported in healthy participants during spontaneous or induced high blood pressure (Saccò et al. 2013). Similarly, unmedicated patients with hypertension report lower pain sensitivity and higher pain thresholds, a condition termed as blood pressure-related hypoalgesia (Olsen et al. 2013; Ottaviani et al. 2018). Several studies have suggested that high blood pressure may protect against clinical pain (Maixner et al. 1997; France \& Katz, 1999; Bruehl et al. 2002; Hagen et al. 2005), entertaining the possibility that the baroreflex - a homeostatic mechanism that controls blood pressure - may also be involved in pain regulation (Suarez-Roca et al. 2019). An association has been also described between blood pressure and the CPM response (Chalaye et al. 2014), supporting the role of baroreceptors in signalling the cardiovascular state of the body to the brain in pain control (Suarez-Roca et al. 2019). Carotid baroreceptor stimulation results in reduced pain sensitivity in both hypertensive and normotensive individuals (Edwards et al. 2003), suggesting that interactions between the ANS and descending pain control systems may involve baroreflex activation (Dayan et al. 2018). Heart rate variability (HRV), an indirect marker of autonomic control of the heart, has also been shown to be related to the perception of pain. Increased low-frequency $\mathrm{HRV}$, a measure specifically linked to baroreflex efficiency
(Moak et al. 2007; Rahman et al. 2011) has been associated with reduced subjective ratings of unpleasantness to cold pain and higher thresholds for low-to moderate levels of thermal pain (Appelhans \& Luecken, 2008). Pain appraisal might play an important role in modulating autonomic responses to pain (Mischkowski et al. 2018), which might in turn explain the association between descending pain modulation and psychological variables such as, for example, pain catastrophising (Traxler et al. 2019). Autonomic measures such as skin conductance and heart rate can predict within-subject perceptual changes in pain and pain ratings (Loggia et al. 2011). This further supports the notion that ANS responses are more than just a nociceptive reflex and that they play a crucial role in the modulation of pain perception.

Perhaps associations between the ANS and pain control are not surprising, given the overlap between brain structures involved in ANS regulation (via the baroreflex) and those involved in pain. For example, the ventro-medial prefrontal cortex (vmPFC), anterior cingulate cortex, thalamus and insula are involved both in the regulation of body autonomic arousal (Benarroch, 2012; Beissner et al. 2013; Sklerov et al. 2018) and in pain perception and elaboration (Peyron et al. 2000; Benarroch, 2006). Animal studies indicate that the periaqueductal grey (PAG) and the rostral ventromedial medulla (RVM) have well-defined roles in descending pain control (Bouhassira et al. 1992; Harris, 1996; Villanueva et al. 1996). In humans, pain control mechanisms have been related to functional networks involving the PAG and the mPFC (Bogdanov et al. 2015) and to resting-state functional connectivity (FC) between the PAG and RVM (Yelle et al. 2009; Harper et al. 2018); but also between the subnucleus reticularis dorsalis and cingulate and dorsolateral prefrontal areas (Youssef et al. 2016). These same brain structures, namely, the cingulate cortex, insula, amygdala and PAG, are also associated with reduced baroreflex sensitivity, particularly the ability of the baroreflex to control transient BP fluctuations in response to physiological stress (Gianaros et al. 2012).

The neurovisceral integration model (Thayer \& Lane, 2000) provides a framework for investigating inter-relationships between descending pain control mechanisms and autonomic functioning in the brain. This model theorises that increased HRV provides an

Elena Makovac completed her PhD in experimental psychology and cognitive neuroscience at the University of Edinburgh. Currently, she works as a postdoctoral research fellow at the Institute of Psychology, Psychiatry and Neuroscience at King's College London. Her main research interest is focused on the understanding of brain-body interactions in healthy volunteers and patients with psychiatric conditions or chronic pain.

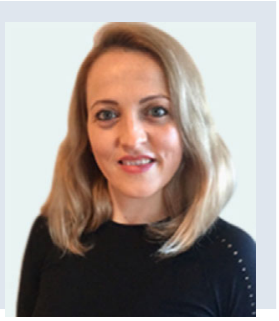


individual with flexibility to adapt in response to changing physiological and environmental demands, governed by a 'central autonomic network' (CAN) (Benarroch, 1993), incorporating the prefrontal cortex (anterior cingulate, insula, orbitofrontal and ventromedial cortex), amygdala, hypothalamus and brain stem (including the PAG and $\mathrm{RVM}$ ). The dynamic and bi-directional interaction between the CAN and the cardiovascular system affects cognition, executive functioning and emotions (Thayer et al. 2009).

Here, we sought to determine brain mechanisms underlying the putative association between descending pain control and autonomic regulation systems in a group of healthy participants. We employed resting-state fMRI (rs-fMRI) and voxel-based morphometry (VBM) as measures of brain function and structure, respectively, focusing our attention on the PAG as a major hub for both autonomic and pain processing. We also examined HRV as an index of autonomic reactivity to pain and performed a behavioural experiment examining descending pain control. We hypothesised that connectivity between the PAG and other pain-related structures would mediate the association between the ANS and descending pain control mechanisms. We also hypothesised an involvement of the RVM, given its role in descending pain controls, as documented by animal studies (Tobaldini et al.2019), and vmPFC in light of recent studies in healthy volunteers and chronic pain patients (Moont et al. 2011; Harper et al. 2018).

\section{Methods}

\section{Ethical approval}

The study was approved by the King's College London Research Ethics Committee (HR-16/17-4769) and was conducted in accordance with the Declaration of Helsinki, except for registration in a database. All participants were informed about the experimental procedures by reading an information sheet. All participants provided written informed consent prior to commencing experimental sessions and were informed that they could withdraw from the experiment at any time.

\section{Participants}

Thirty healthy participants took part in the CPM experiment (12 women, 18 men, mean age across the group $=26.4 ; \mathrm{SD}=4.7$ years). Out of these 30 participants, 18 (6 women, 12 men, mean age across the group $=27.1 ; \mathrm{SD}=5.1$ years) took part in the CPM, MRI and physiology experimental sessions (detailed below). The 18 participants who took part in the MRI study are a subset of a larger group, in whom the relationship between rs-fMRI and HRV has been described elsewhere (Hohenschurz-Schmidt et al. 2020). Therefore, 30 participants in total were considered for the behavioural (CPM) analyses whilst 18 participants only were considered for the regression analysis with MRI and HRV data. All participants were right-handed as assessed by the Edinburgh handedness inventory (Oldfield, 1971). Exclusion criteria included MRI contraindications, history of brain injuries, hypertension, neurological or psychiatric disease, and alcohol or drug abuse. To minimise the potential effects of menstrual cycle-related hormone fluctuations on pain responses (Vincent \& Tracey, 2010) and descending pain modulating effects (Tousignant-Laflamme \& Marchand, 2009), female participants were all tested within the follicular phase of their menstrual cycle. Furthermore, to minimise the influence of diurnal variations on pain responses (Strian et al. 1989) and on rs-fMRI networks activity (Jiang et al. 2016), participants were always tested at the same time during the day across the three experimental sessions. At the beginning of each visit, participants were tested for drug use (urine drug test) and alcohol consumption (alcohol breathalyser).

\section{Experimental procedure}

Participants took part in one familiarisation session, one scanning session and one post-scanning CPM session. At the beginning of each experimental session, participants completed the 'state' subscale of the State-Trait Anxiety Inventory (STAI) (Spielberger et al. 1983), in order to evaluate for possible differences in anxiety levels between the three testing sessions.

\section{Familiarisation and thresholding session}

During the familiarisation session, participants became accustomed to the neuroimaging environment (in a simulated 'mock' scanner), and with the cold-pain and pressure-pain stimulation protocol. The cold pain stimulation was delivered via an aluminium probe $(4 \times 20 \mathrm{~cm})$, attached to the volar surface of their right forearm, through which cold water $\left(2^{\circ} \mathrm{C}\right)$ was constantly circulated by means of two chillers.

Next, participants were individually thresholded for pressure pain. A laboratory-built pressure-inducing thumb device was used to apply pressure on the right-side thumbnail. Participants received an ascending series of pressure stimuli and were asked to state when they felt the first sensation of pain (pain threshold) and when they reached a 70 on a visual analogue scale (VAS) from 0 (no pain) to 100 (maximum pain tolerance). Three intermediate pressure values between these thresholds were created to provide five equally spaced pressure values, starting with the threshold and ending with the highest values corresponding to each individuals' VAS 70/100. For 
example, if the minimum pain threshold was represented by a pressure of $200 \mathrm{kPa}$ in the ascending series and the maximum pain threshold of $>70$ was reached with a pressure of $600 \mathrm{kPa}$, the randomised series would consist of pressures of 200, 300, 400, 500 and $600 \mathrm{kPa}$. The five pressure values were then presented to the participant in a random order, each repeated three-times (total number of new stimulations $n=15$ ). After each stimulation, the participant was required to give a rating on a VAS scale. Based on the 15 pressure values and 15 subjective pain ratings, a polynomial regression function was used to determine each individual's representation of VAS 50, 60 and 70 (Kosek et al. 2017). These values were then used in our CPM protocol (third session).

\section{MRI scanning session and physiological recording}

During the scanning sessions, participants underwent three fMRI scans, each of $6 \mathrm{~min}$ in duration: a baseline; a cold-pain and a post-cold recovery block. The investigation of the post-cold recovery phase aimed at identifying pain-related carry over effects and the results from this analysis have been reported elsewhere (Makovac et al. 2019). A multi-echo resting-state fMRI (rs-fMRI) approach was adopted to mitigate problems related to pain-induced movements, which are an important source of non-BOLD artefacts in pain studies (Siegel et al. 2016; Dipasquale et al. 2017; Moayedi et al. 2018). During each period, participants were instructed to keep still with their eyes open, focusing on a fixation cross presented at the centre of the screen, without thinking of anything or falling asleep.

MRI images were acquired on a GE MR750 scanner, equipped with a 32-channel receive-only head coil (NovaMedical). Structural volumes were obtained using a high-resolution three-dimensional magnetization-prepared rapid gradient-echo sequence (TR = $7312 \mathrm{~ms}, \mathrm{TE}=3.02 \mathrm{~ms}$, flip angle $=11^{0}$, slice thickness $=$ $12 \mathrm{~mm}, 196$ sagittal slices, FOV $=270 \mathrm{~mm}$ ). Multi-echo rs-fMRI datasets were acquired using a T2*-weighted blood oxygenation level dependent (BOLD) sensitive echo planar imaging (EPI) sequence $(\mathrm{TR}=2 \mathrm{~s}, \mathrm{TE} 1=$ $12 \mathrm{~ms}$, TE2 $=28 \mathrm{~ms}$; TE3 $=44 \mathrm{~ms}$; flip-angle $80^{\circ}, 32$ slices, $3 \mathrm{~mm}$ slice thickness, $240 \mathrm{~mm}$ FOV, voxel size $3.75 \times 3.75 \times 3 \mathrm{~mm}$ ). During rs-fMRI, heart rate was monitored using an inbuilt MRI-compatible finger pulse oximeter (General Electric) recorded digitally from an analogue output waveform at a sample rate of $40 \mathrm{~Hz}$, fitted to the participants' dominant right index finger.

\section{CPM session}

Participants were tested during a third session (on a different day from the scanning session), where they underwent a CPM protocol, in order to test descending pain modulatory mechanisms.

\section{CPM experimental protocol}

During CPM, short-duration noxious pressure test stimuli were delivered to the right-hand thumbnail prior to, simultaneously with, and immediately following a noxious cold conditioning stimulus applied to the left-hand forearm. The intensity of the pressure test stimuli was determined individually for each participant, based on the previously described thresholding procedure, to synthesise pressures to induce pain ratings of 50, 60 and 70 on a $0-100$ VAS. Given the lack of unanimous consensus regarding the optimal psychophysical characteristics of the test stimulus and conditioning stimulus in eliciting the CPM effect, we randomly delivered pressure pain stimuli corresponding to the three subjective intensities $(50,60$ and 70 VAS), to explore the effect of test stimulus intensity on the CPM response. To account for potential carry-over effects of the conditioning stimulus, test stimuli were presented simultaneously with the conditioning stimulus, and for 6 min following the conditioning stimulus.

Participants underwent three separate experimental runs (refer to Fig. 1): (i) test stimuli only (Baseline condition), and two identical CPM runs (CPM RUN1 and CPM RUN2; Fig. 1), separated by a 15 min break. CPM investigations were split into two runs to provide an adequate number of events per experimental condition but to minimise response habituation and/or peripheral sensitisation. The CPM runs comprised two $6 \mathrm{~min}$ mini-blocks, the first during which cold stimulation was present (CPM Cold-ON condition), the second without cold stimulation (CPM Cold-OFF condition). During the Baseline condition, participants rated the intensity of pain evoked by pressure pain stimuli, on a computerized VAS ranging from 0 (no pain) to 100 (maximal imaginable pain). Twenty-four pressure test stimuli were delivered in a random order to their right thumbnail, eight per class of stimulus intensity (50, 60 and 70 VAS). Each test stimulus had a duration of $2 \mathrm{~s}$, followed by an $6 \mathrm{~s}$ VAS rating period then $5 \mathrm{~s}$ inter-trial interval. The total duration of the Baseline run was $9.2 \mathrm{~min}$. During the CPM run (CPM RUN1 and CPM RUN2), participants were instructed to rate the pressure pain test stimuli (as per the Baseline condition), and not the painful cold conditioning stimulus applied to their left forearm. Finally, participants subjectively rated the intensity of the painful cold conditioning stimulus using a computerized VAS ranging from 0 (no pain) to 100 (maximal imaginable pain).

\section{Statistical analysis}

All data are expressed as mean and SD. Normality of the variables was examined using Shapiro-Wilks tests. 
Non-normally distributed variables were logarithmically transformed before proceeding with further analyses. The Benjamini-Hochberg procedure was used for multiple-comparisons correction (Thissen et al. 2002).

\section{State anxiety}

A one-way within-subject ANOVA was used to explore for differences in state anxiety among the three experimental sessions (thresholding, scanning session, CPM session). Possible associations between state anxiety, HRV and CPM were investigated by means of Pearson's correlation coefficient. Statistical threshold was set at $P<0.05$.

\section{CPM analysis}

CPM Cold-ON and CPM Cold-OFF conditions were obtained by averaging pressure ratings from CPM RUN1 and CPM RUN2 (Fig. 1). Raw data from the separate runs are shown in Table 1. $\triangle$ CPM Cold-ON and $\triangle \mathrm{CPM}$ Cold-OFF scores were calculated by subtracting Baseline VAS rating values from the CPM Cold-ON and CPM Cold-OFF conditions ratings, respectively. Negative $\triangle \mathrm{CPM}$ values indicated a decrease in perceived pressure pain intensity during the conditioning stimulus $(\triangle \mathrm{CPM}$ Cold-ON) or following the conditioning stimulus ( $\triangle \mathrm{CPM}$ Cold-OFF), whereas positive values indicated an increase in perceived pain. A two-way within-subject ANOVA was used to explore the main effect of pressure (50, 60 and 70 VAS), condition (Baseline, CPM Cold-ON, CPM Cold-OFF), and the pressure $\times$ condition interaction.
Table 1. Mean and standard deviation for pain rating in the five experimental conditions and three pressure pain conditions (corresponding to the individual VAS of $50 / 100,60 / 100$, and 70/100)

\begin{tabular}{llll}
\hline & \multicolumn{3}{c}{ Pressure levels } \\
\cline { 2 - 4 } & 50 & 60 & 70 \\
\hline Pressure only & $31.5(17.2)$ & $38.3(16.9)$ & $45.3(18.8)$ \\
CPM Cold-ON RUN1 & $29.7(20.3)$ & $36.3(19.7)$ & $41.0(17.8)$ \\
CPM Cold-ON RUN2 & $34.3(20.6)$ & $41.8(21.2)$ & $45.6(20.9)$ \\
CPM Cold-OFF RUN1 & $36.2(17.2)$ & $41.4(20.2)$ & $46.7(20.0)$ \\
CPM Cold-OFF RUN2 & $40.5(19.7)$ & $42.1(22.3)$ & $49.3(20.0)$ \\
\hline
\end{tabular}

Data obtained from $n=30$ participants.

\section{Heart rate variability analysis}

Inter-beat interval values were visually inspected, and potential artefacts were manually removed. Inter-beat-intervals were then entered into Kubios HRV Standard ver. 3.0.2 (Tarvainen et al. 2014) and further artefact correction was applied using the lowest correction threshold appropriate for individual artefact severity. To capture the most salient autonomic response to pain (without the interfering effect of habituation), low-frequency HRV (LF-HRV; 0.04-0.15 Hz) was derived from recordings of the initial $3 \mathrm{~min}$ during cold-pain application. LF-HRV is a measure of interest in acute pain studies as it is an index of baroreflex activity (Goldstein et al. 2011; Rahman et al. 2011). Recent meta-analytic works have highlighted the influence of acute experimental pain on LF-HRV (Koenig et al. 2014). Next, LF-HRV data were normalised. Normalised units (n.u.) of HRV spectral data express the relative value

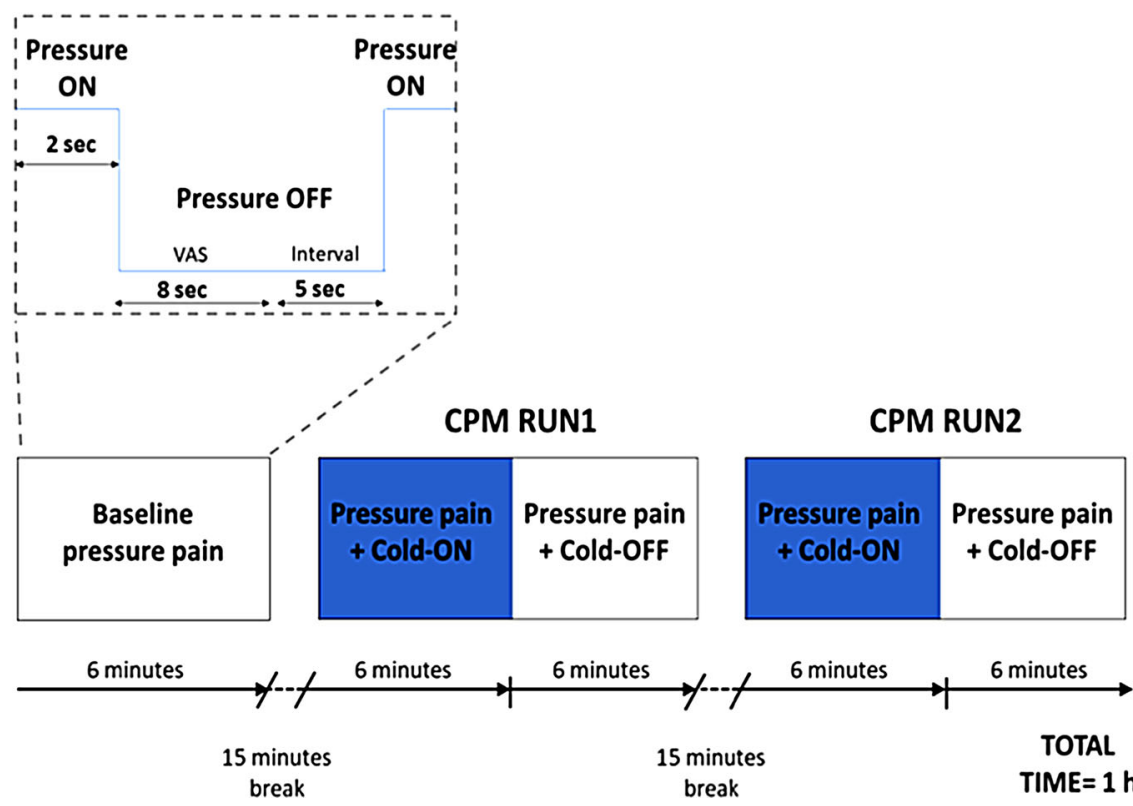

Figure 1. A graphical representation of the experimental protocol

Participants were required to rate $2 \mathrm{~s}$ painful pressure stimuli for 6 min (Baseline condition), followed by a 15 min break. During CPM, participants rated pressure pain with a simultaneous contralateral cold stimulation for 6 min (CPM Cold-ON condition), followed by ratings of pressure pain without the contralateral cold (CPM Cold-OFF condition). 
of each frequency component in relation to the total power spectrum minus the very low frequency band (VLF): LF-HRVnu $=\mathrm{LF}\left(\mathrm{ms}^{2}\right) /\left(\right.$ total power $\left(\mathrm{ms}^{2}\right)-\mathrm{VLF}$ $\left(\mathrm{ms}^{2}\right)$ (Tarvainen et al. 2014). Raw (non-normalised), LF-HRV reflects an influence of both the sympathetic and parasympathetic nervous systems (Reyes del Paso et al. 2013). Nevertheless, in the case of normalized units (necessary in studies with a small sample size) LF (and high frequency-HF) normalized measures become linearly associated, and therefore provide a more generalized index of autonomic reactivity (Burr, 2007). A time-based HRV measure - the root mean square successive difference, rMSSD - was also calculated. RMSSD is an HRV measure which is sensitive to changes in parasympathetic tone (Laborde et al. 2017), providing complementary information to LF-HRV analyses.

\section{Rs-fMRI pre-processing}

Analysis of Functional NeuroImages (AFNI, Cox, 1996), the Advanced Normalization Tools (ANTs) (Avants et al. 2011) and the FMRIB Software Library (FSL, Smith et al. 2004) toolboxes were used to process multi-echo rs-fMRI data. The volumes were first re-aligned and slice timing-corrected. Next, multi-echo sequences were optimally combined (OC) by taking a weighted summation of the three echoes using an exponential T2* weighting approach (Posse et al. 1999). A multi-Echo ICA approach (implemented by the tool meica.py, Version v2.5 beta9) (Kundu et al. 2013) was adopted to de-noise the OC-data. Multi-Echo ICA is an effective method for the removal of physiological and motion-related noise, resulting in a significant increase in the temporal SNR (Kundu et al. 2013; Dipasquale et al. 2017). Briefly, multi-echo principal component analysis was first used to reduce the data dimensionality in the OC dataset. Spatial ICA was then applied on one echo, and the independent component time-series were fitted to the pre-processed time-series from each of the three echoes to generate ICA weights for each echo. These weights were then fitted to the linear TE-dependence and TE-independence models to generate $F$-statistics and component-level $\kappa$ and $\rho$ values, which respectively indicate BOLD and non-BOLD weightings. The $\kappa$ and $\rho$ metrics were then used to identify non-BOLD-like components to be regressed out of the OC dataset as noise. For further technical details on ME-ICA see Kundu et al. (2015). Lastly, the white matter (WM) and cerebrospinal fluid (CSF) signals were regressed-using FSL. A high-pass temporal filter (with a cut-off frequency of $0.005 \mathrm{~Hz}$ ) and spatial smoothing with a $5 \mathrm{~mm}$ full-width at half-maximum (FWHM) Gaussian kernel were then applied. Each participant's dataset was co-registered to its corresponding structural scan with an affine registration and normalised to standard MNI152 space (with a non-linear approach) with a $2 \times 2 \times 2 \mathrm{~mm}^{3}$ resampling using ANTs.

\section{VBM pre-processing}

T1-weighted volumes from all participants were visually reviewed to exclude the presence of macroscopic artefacts, prior to pre-processing in SPM12 (Statistical Parametrical Mapping, http://www.fil.ion.ucl.ac.uk/spm/) using the DARTEL algorithm. In brief, the MR images were bias-field corrected to correct non-uniformities then segmented into grey matter (GM), white matter and cerebro-spinal fluid sections using a priori tissue probability maps in International Consortium of Brain Mapping (ICBM) template space. The individual GM images were subsequently normalized to the Montreal Neurological Institute (MNI) template with a $1.5 \times 1.5 \times 1.5 \mathrm{~mm}^{3}$ voxel size and modulated to maintain original individual GM volumes. Finally, all GM images were smoothed with $5 \mathrm{~mm}$ FWHM isotropic Gaussian kernel. Total intra-cranial volume (ICV), age and gender were used as covariate of no interest in subsequent GLM analyses (see VBM Analyses).

\section{Seed-based fMRI: first level analysis}

An ROI for the PAG (a $3 \mathrm{~mm}$-radius sphere; $\mathrm{MNI}=0$, $-32,-10$; Coulombe et al. 2017) was constructed using the Marsbar toolbox implemented in SPM 12 (http:// marsbar.sourceforge.net/). The average resting state fMRI time-series for the ROI was extracted from smoothed images for each participant and scan and used as a regressor in a 1st level SPM analysis, to explore the network of areas associated with the seed region.

Assessment of relationships between brain FC, brain structure and self-reported pain and HRV measures

For both seed-based fMRI and VBM analyses, we sought to determine brain regions positively or negatively associated with $\triangle \mathrm{CPM}$ Cold-ON, with LF-HRVnu in response to cold, or with the interaction between the two variables (i.e. the interaction term obtained by multiplying $\triangle \mathrm{CPM}$ Cold-ON with LF-HRVnu). We further explored the association between brain structure/FC and both pressure pain threshold and cold pain ratings to determine whether our results were dependent on descending pain modulating pathway or rather on more general characteristics of the stimuli (i.e. pressure pain threshold or subjective perception of cold pain) adopted in our protocol.

Seed-based second level analyses. For the second-level seed-based regression analyses, we calculated the $\Delta \mathrm{FC}$ of the PAG network in response to cold pain, by 
subtracting baseline FC maps from those during cold pain. $\triangle F C$ maps were then used for regression analyses within the framework of the general linear model with $\triangle \mathrm{CPM}, \mathrm{LF}-\mathrm{HRVnu}$, and the $\triangle \mathrm{CPM} \times \mathrm{LF}-\mathrm{HRVnu}$ interaction term as covariates of interest; these analyses were performed to explore brain areas in which FC during pain was associated with descending pain modulation and with the interaction between descending pain modulation and autonomic reactivity to pain. Age and gender were used as covariates of no interest.

VBM second level analyses. Statistical analyses were performed on smoothed GM maps within the framework of the general linear model. As in the seed-based fMRI analysis, $\triangle \mathrm{CPM}$, LF-HRVnu (during cold) and the $\triangle \mathrm{CPM} \times \mathrm{LF}-\mathrm{HRVnu}$ interaction term were used as variables of interest in regression analyses, whereas intra-cranial-volume volume was used as a nuisance covariate to consider potential confounding effects of individual differences in head size.

Small volume correction analyses for a priorihypothesized regions of interest. To test a priori hypotheses, small volume correction (SVC) analyses were performed in the vmPFC, PAG (for the structural analyses only) and rostral ventromedial medulla (RVM). A $5 \mathrm{~mm}$ radius spherical mask was created for the PAG $(\mathrm{MNI}=0,-32,-10)$. For the RVM, three separate $3 \mathrm{~mm}$ radius spheres were created, centred at MNI $(x, y)=$ $[0,-34]$, at three contiguous rostro-caudal levels from
MNI $(z)=[-53$ to -49$]$, as described in Marciszewski et al. (2018). The three contiguous spheres were then merged resulting in a final single ROI. Finally, an anatomical mask was defined for the vmPFC, derived from the SPM-12 anatomy toolbox (Eickhoff et al. 2005). A graphical representation of the three ROIs is provided Fig. 2.

For each participant, we extracted GM volume values and FC parameter estimates from the vmPFC, PAG and RVM masks. These values were used for further mediation analyses. GM volume values and FC parameter estimates were also extracted from significant voxels and plotted for illustrative purposes (and to explore for possible outliers). Parameter estimates from significant voxels were also used for additional correlation analysis, to investigate whether $\triangle \mathrm{CPM}$ related brain areas were associated with LF-HRVnu and vice versa.

Statistical inference. For both types of analyses, statistical thresholds were set to $P<0.05-$ FWE-corrected at cluster level (Worsley \& Friston, 1995) (cluster size defined using uncorrected voxel-level threshold $P<0.001$ ). Exploratory analyses were also performed using a less stringent cluster forming threshold $(P<0.005)$.

\section{Correlation and mediation analyses}

Mediation analysis was performed to ascertain whether pain and ANS related brain regions mediated the relationship between autonomic reactivity to pain and

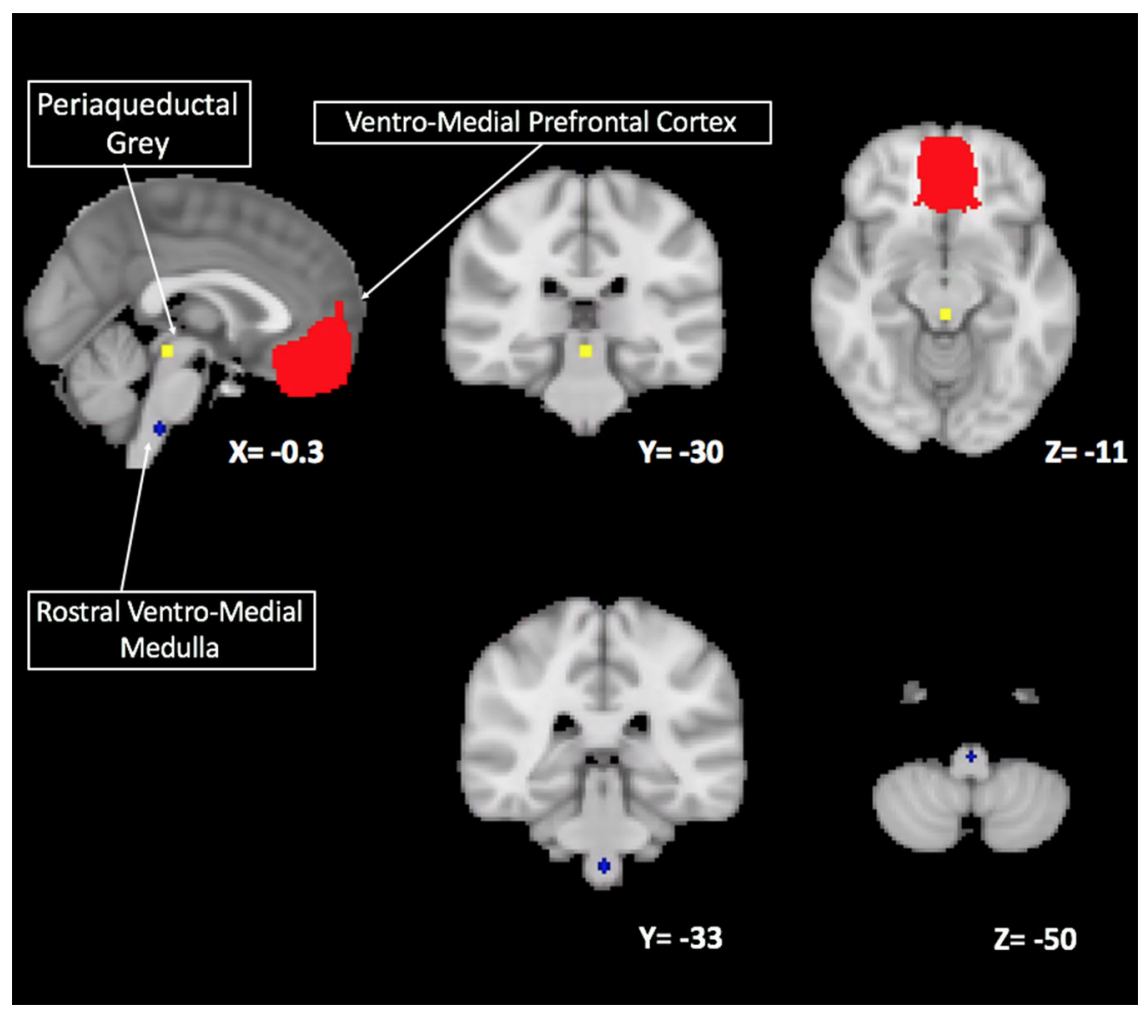

Figure 2. Graphical representation of the regions of interest (ROIs) adopted for the small volume correction 
CPM response. Data analysis was performed with SPSS 22.0 for Windows (SPSS Inc, USA). First, Pearson's correlation coefficient was used to explore the association between LF-HRVnu during cold and $\triangle \mathrm{CPM}$ ratings $(\triangle \mathrm{CPM}=$ (pressure + cold $)-$ (pressure only baseline)). Next, the mean parameter estimates were extracted from SVC masks (PAG, RVM and vmPFC) were entered in a mediation analysis using PROCESS (Hayes, 2012) in IBM SPSS version 23.0 (IBM Corp., Armonk, NY). LF-HRVnu was introduced as an independent variable, $\triangle \mathrm{CPM}$ was the outcome variable, and brain functional and structural parameter estimates from SVC masks were possible mediators. The indirect effect was tested with 20,000 percentile-corrected bootstrap confidence intervals $(95 \%)$.

\section{Results}

\section{Subjective pain ratings following cold pain}

Following 6 min cold stimulation, participants gave an average pain rating of $44.94(\mathrm{SD}=21.82)$ on a $0-100 \mathrm{VAS}$ scale. At the end of the 6 min post-cold pain recovery phase, the participant pain intensity (rated on the VAS) was $1.50(\mathrm{SD}=3.37)$.

\section{Effect of anxiety}

We did not observe a significant difference in state anxiety between the three experimental sessions $(F(2,48)=1.03$, $P=0.37)$.

\section{VAS ratings during CPM}

Mean and standard deviation for pain rating in the five experimental conditions (Baseline condition, CPM RUN1 Cold-ON and Cold-OFF, CPM RUN2 Cold-ON, and Cold-OFF) and three pressure pain conditions (VAS $50 / 100,60 / 100$ and 70/100) are reported in Table 1.

Ratings from conditions CPM RUN1 and CPM RUN2 were averaged, separately for Cold-ON and Cold-OFF. We computed a within-subject ANOVA analysis with two factors (pressure $(50,60,70$ VAS)) and (condition (Baseline, Cold-ON, Cold-OFF)). Logarithmic transformation was applied to achieve normality of the data. A main effect of pressure was found $(F(2,58)=31.02$, $P=0.001)$, driven by higher VAS ratings in response to 60-pressure stimuli comparing to 50-pressure stimuli $($ mean $(\mathrm{SD})=31.5$ (5.8) vs. 38.3 (16.9) for 50 and 60 pressure respectively, $t(20)=6.92, P=0.001)$; higher ratings to 70 -pressure stimuli (mean $(\mathrm{SD})=45.8(18.2)$ ) when compared to both 60-pressure (mean $(\mathrm{SD})=40.0$ $(18.2) ; t(29)=5.87, P=0.001)$ and 50-pressure stimulations (mean $(\mathrm{SD})=34.3(17.5) ; t(29)=8.27$,
$P=0.001)$. A main effect of Condition was also observed $(F(2,58)=4.84, P=0.016)$, driven by an increase in overall perceived pain intensity in the CPM Cold-OFF condition (mean $(\mathrm{SD})=42.8$ (19.8)) when compared to Baseline (mean $(\mathrm{SD})=38.4(17.3), t(29)=2.43 P=0.02)$ and CPM Cold-ON trials (mean (SD) $=38.9$ (17.9), $t(29)=3.0 P=0.005)$. No significant difference was observed when comparing CPM to Baseline $(t(29)=0.33$, $P=0.74)$. No significant effect of the condition $\times$ pressure interaction was observed $(F(2,58)=2.01, P=0.12)$. Give the lack of significant interaction with pressure levels, data were averaged across different pressure conditions for further analyses.

The distribution of $\triangle \mathrm{CPM}$ (CPM Cold-ON and CPM Cold-OFF) values is represented in Fig. 3. During the CPM Cold-ON condition, 16 participants reported an increase in pain rating (i.e. positive $\triangle \mathrm{CPM}$ ), and 14 participants reported a decrease in pain ratings (i.e. a negative $\triangle \mathrm{CPM})$. During the CPM Cold-OFF condition, 22 participants reported a positive $\triangle \mathrm{CPM}$, whereas 8 participants reported a negative $\triangle \mathrm{CPM}$.

\section{Exploratory correlation analyses}

Exploratory analyses were performed to investigate whether the magnitude of the CPM response was related to either the characteristics of the test stimulus, the conditioning stimulus, or to participants' anxiety levels. Results indicated that the magnitude of the CPM Cold-ON response ( $\triangle \mathrm{CPM}$ Cold-ON) was not associated with cold pain ratings $(P=0.92)$, with individual pressure pain thresholds $(P=0.81)$, or state anxiety levels during the CPM session $(P=0.83)$.

\section{Correlation between HRV and CPM response}

We observed a negative correlation between LF-HRVnu during cold pain and $\triangle$ CPM Cold-ON $(r=-0.49$, $P=0.041$ ), where individuals with the highest increase in LF-HRVnu during cold pain exhibited the strongest decrease in pressure pain rating during CPM (Fig. 4). We did not observe a significant association between baseline LF-HRVnu and $\triangle$ CPM Cold-OFF $(P=0.17)$. Similarly, we did not observe a significant correlation between RMSSD and both $\triangle$ CPM Cold-ON $(P=0.65)$ and $\triangle \mathrm{CPM}$ Cold-OFF $(P=0.14)$. Given the lack of correlation between RMSSD and CPM, further associations between this HRV measure and brain structure and functional networks were not pursued.

\section{Seed-based analysis}

Seed-based FC analysis of the PAG showed a network comprised of the bilateral parahippocampal/amygdalar 
Table 2. Associations between CPM scores, autonomic reactivity during pain, functional connectivity and brain structure, investigated in 18 participants

\begin{tabular}{|c|c|c|c|c|c|}
\hline \multirow[b]{2}{*}{ Brain area } & \multirow{2}{*}{$\begin{array}{l}\text { Direction of } \\
\text { correlation }\end{array}$} & \multicolumn{2}{|c|}{ Cluster } & \multicolumn{2}{|c|}{ Voxel } \\
\hline & & $K$ & $p$ (FWE) & $T$ & MNI $(x, y, z)$ \\
\hline \multicolumn{6}{|c|}{ A. Rs-fMRI regression analyses } \\
\hline \multicolumn{6}{|c|}{ Association between FC and $\triangle \mathrm{CPM}$ (Cold ON) } \\
\hline Precuneus, left & Positive & 175 & 0.043 & 8.05 & $-22,-64,28$ \\
\hline Anterior supramarginal gyrus, right & Positive & 278 & 0.006 & 4.97 & $-44,-32,40$ \\
\hline vmPFC & Negative & 445 & $0.051 *$ & 4.24 & $-14,52,6$ \\
\hline Rostral ventro-medial medulla & Negative & 714 & $0.005 *$ & 6.95 & $-6,-32,-36$ \\
\hline \multicolumn{6}{|c|}{ Association between FC and LF-HRV during cold pain } \\
\hline Angular gyrus, right & Negative & 930 & $0.001 *$ & 5.24 & $66,-16,-24$ \\
\hline \multicolumn{6}{|l|}{ B. VBM regression analyses } \\
\hline \multicolumn{6}{|c|}{ Association between GM volume and $\triangle \mathrm{CPM}$ (Cold ON) } \\
\hline vmPFC & Negative & 178 & $0.001^{\#}$ & 3.80 & $0,38,-10$ \\
\hline
\end{tabular}

$\mathrm{vmPFC}=$ ventro-medial prefrontal cortex; PAG = periaqueductal grey.

*Results obtained with a cluster forming threshold $P<0.005$;

\# results obtained with a small volume correction.

areas, middle cingulate cortex, anterior insular cortex and the vmPFC, in line with other published studies (see Makovac et al. 2019).

\section{Associations between PAG functional connectivity, $\triangle \mathrm{CPM}$ and autonomic reactivity (LF-HRVnu) during cold pain}

Association between PAG FC and $\triangle$ CPM Cold-ON, LF-HRVnu and $\triangle$ CPM Cold-ON $\times$ LF-HRVnu interaction. A positive association was observed between $\triangle \mathrm{CPM}$ scores and $\triangle \mathrm{FC}$ in response to cold pain between the PAG and the supramarginal gyrus and left pre- cuneus. At a more liberal cluster forming threshold (cluster forming threshold $P<0.005$ ) we also observed a negative association between $\triangle \mathrm{CPM}$ Cold-ON and the PAG $\triangle F C$ in response to pain, with both the vmPFC and RVM (extending to the cerebellum) (Fig. 5A and Table 2). This result indicates that individuals with more efficient descending pain inhibition (as indexed by negative $\triangle \mathrm{CPM}$ values) had a lower increase in FC between the PAG and more posterior parietal areas and stronger increase in FC between the PAG and the vmPFC and RVM during cold pain. At a more liberal cluster forming threshold $(P<0.005)$, we observed a negative association between LF-HRVnu and the $\triangle \mathrm{FC}$ between PAG and right angular gyrus (Fig. 5A and Table 2). We

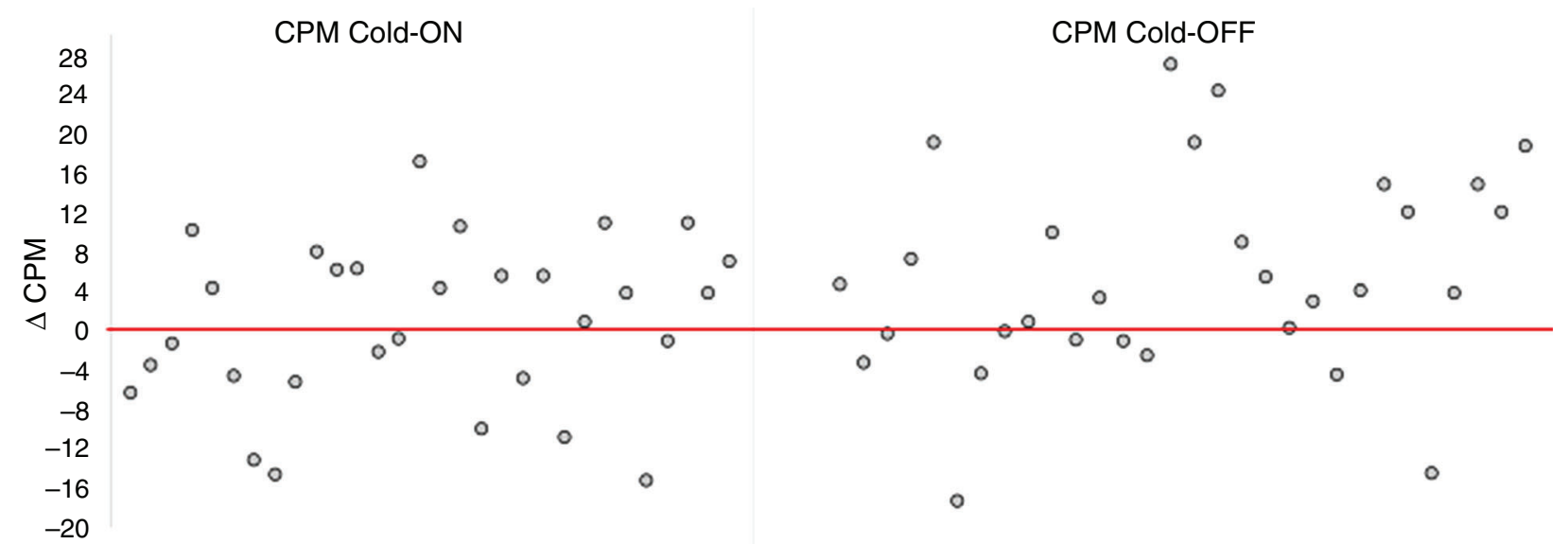

Figure 3. Conditioned pain modulation behavioural results in $\mathbf{3 0}$ participants

The magnitude of the CPM Cold-ON effect ( $\triangle$ CPM Cold-ON) and CPM Cold-OFF effect ( $\triangle$ CPM Cold-OFF) was defined as the difference in pressure pain rating between baseline (pressure only) and the CPM Cold-ON condition (cold conditioning stimulus simultaneously presented with pressure pain test stimulus) or CPM Cold-OFF condition (pressure pain test stimulus presented immediately following the cold conditioning stimulus). 
did not observe any association between PAG FC and the $\triangle \mathrm{CPM}$ Cold-ON $\times$ LF-HRVnu interaction term.

Exploratory analyses of the association between PAG FC and pressure pain threshold and cold-pain ratings. We did not observe any positive or negative association between PAG $\triangle \mathrm{FC}$ and baseline pressure pain ratings.

\section{Associations between brain structure, CPM score and} autonomic reactivity (LF-HRVnu) during cold pain

We observed a negative correlation between $\triangle \mathrm{CPM}$ Cold-ON score and vmPFC volume (Fig. 5B). These results indicate that individuals with negative $\triangle \mathrm{CPM}$ values exhibited larger vmPFC GM volumes. We did not observe any association between GM volume and LF-HRVnu during cold, nor between GM volume and the $\triangle \mathrm{CPM} \times$ LF-HRVnu interaction term.

Exploratory analyses of the association between GM volume and pressure pain threshold and cold-pain ratings. We did not observe any positive or negative association between GM volume and baseline pressure pain ratings.

\section{Correlation between CPM associated PAG functional connectivity estimates, grey matter volume and LF-HRVnu}

We performed post hoc correlation analyses to explore associations between CPM related brain areas and

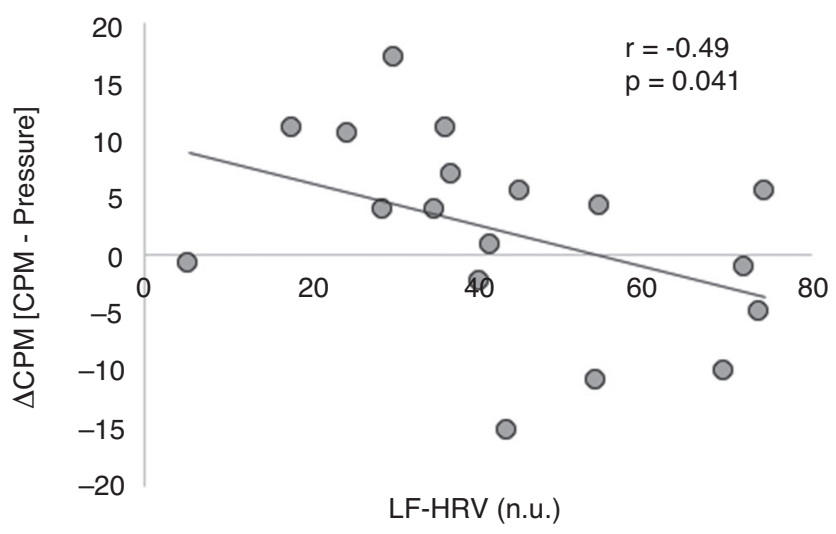

Figure 4. The association between $\triangle C P M$ Cold-ON and LF-HRVnu, investigated in 18 participants using Pearson's correlation coefficient

A negative correlation between $\triangle$ CPM Cold-ON (CPM Cold - ON-Baseline pressure pain) and LF-HRVnu was observed, indicating that individuals with the highest LF-HRVnu during cold pain exhibited a decrease in pressure pain rating during simultaneous contralateral cold pain. n.u. = normalized units.
LF-HRVnu. We observed a positive correlation between the strength of PAG-vmPFC FC with LF-HRVnu, indicating that individuals with the highest increase in LF-HRVnu during pain had also the strongest FC among these the PAG and the vmPFC when engaged in descending pain modulation (Fig. 6).

\section{Mediation analysis}

We observed a mediating role of PAG-vmPFC FC in the relationship between LF-HRVnu and the CPM effect. The total effect of LF-HRVnu on $\triangle \mathrm{CPM}$ was significant (path $\mathrm{C} ; t=-2.23, P=0.041$ ). Adding PAG-vmPFC FC as the mediator, the direct effect of LF-HRV on $\triangle \mathrm{CPM}$ was no longer significant (path $\mathrm{C}^{\prime}$; $t<1, P=0.31$ ), whereas the indirect path via PAG-mPFC FC was significant $(95 \% \mathrm{CI}$ $[-0.27,-0.08])$. Therefore, the effect of LF-HRVnu on $\triangle \mathrm{CPM}$ was largely mediated by PAG-vmPFC FC (Fig. 7).

\section{Discussion}

ANS activity can influence pain perception (Saccò et al. 2013). This interaction might involve descending pain modulatory pathways. In this healthy volunteer study, we used rs-fMRI and VBM to investigate brain areas underlying one's ability to engage descending pain modulatory mechanisms (as measured by CPM) and autonomic reactivity to cold pain (as measured by the LF-HRVnu index). We observed an association between LF-HRVnu during cold pain and the magnitude of an individual's CPM response. The CPM response was associated with PAG-PCC, PAG-vmPFC and PAG-RVM FC, and with PAG and vmPFC GM volume. The same brain areas were also associated with LF-HRVnu during cold pain. The strength of the association between CPM and LF-HRVnu was mediated by PAG-vmPFC FC in response to tonic cold pain. Our results provide evidence for the mechanisms through which central, peripheral and pain systems interact, indicating that an adaptive autonomic reaction to pain is involved in descending pain inhibition.

We have demonstrated an association between each person's autonomic reactivity to pain and the efficiency of their descending pain control systems, namely a negative association between an LF-HRVnu during tonic cold pain and a decrease in subjective ratings of pressure pain during simultaneous application of a cold pain. Our findings accord with one previous study in patients with fibromyalgia showing that systolic blood pressure responses during the cold pressor test are positively associated with the CPM inhibiting effect, suggesting that chronic pain might be linked to a reduced blood pressure response to the conditioning stimulus during CPM (Chayale et al. 2014). Our study, in healthy participants, supports the existence of a functional pain 


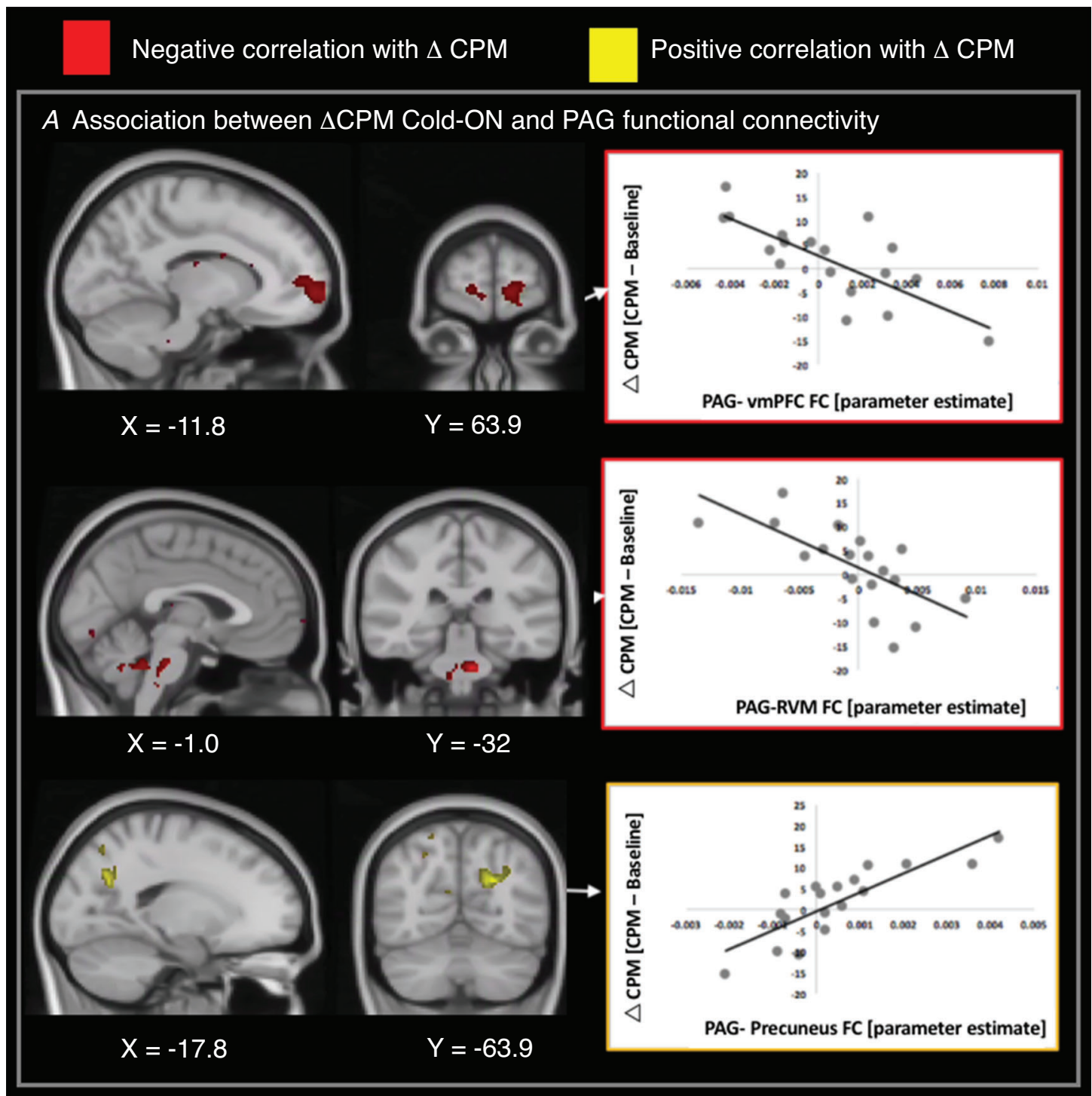

\section{$B$ Association between $\triangle \mathrm{CPM}$ Cold-ON and brain structure}

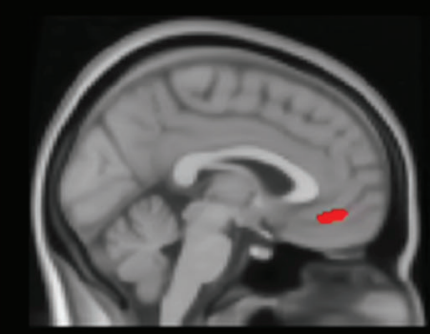

$X=-1.0$

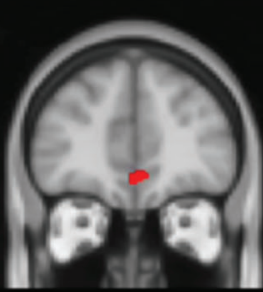

$\mathrm{Y}=41.0$

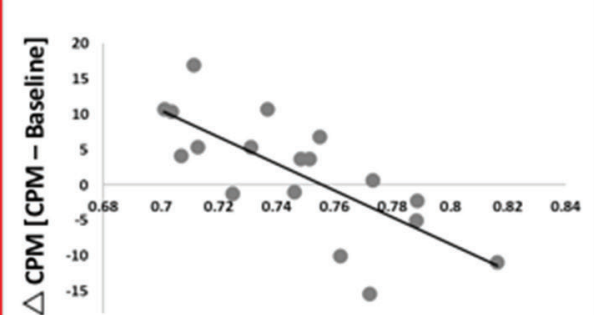

vmPFC GM volume [parameter estimate]

Fure 5. Association between CPM and brain structure and function, explored in 18 participants

$A$, functional connectivity between the PAG and both the VMPFC and RVM was negatively associated with $\triangle$ CPM Cold-ON (CPM Cold-ON-Baseline pressure). B, $\triangle$ CPM Cold-ON was negatively associated with vmPFC GM volume. 
inhibitory feedback system described in animal models that is responsible for blood pressure-related hypoalgesia (Ghione, 1996). This model describes a reflexive increase in sympathetic arousal during pain, resulting in an increase in blood pressure (Reis et al. 1989). The increase in blood pressure stimulates baroreceptors, whose afferent information activates descending pain modulatory pathways, modulating perceived pain, and regaining homeostasis (Bruehl \& Chung, 2004). In this work, we focused our interest on the relationship between ANS reactivity to a tonic cold painful stimulus and CPM response. However, both test and conditioning stimuli are likely to produce acute ANS reactions. Future studies should consider not only ANS reactions to conditioning stimuli, and test stimuli in isolation, but

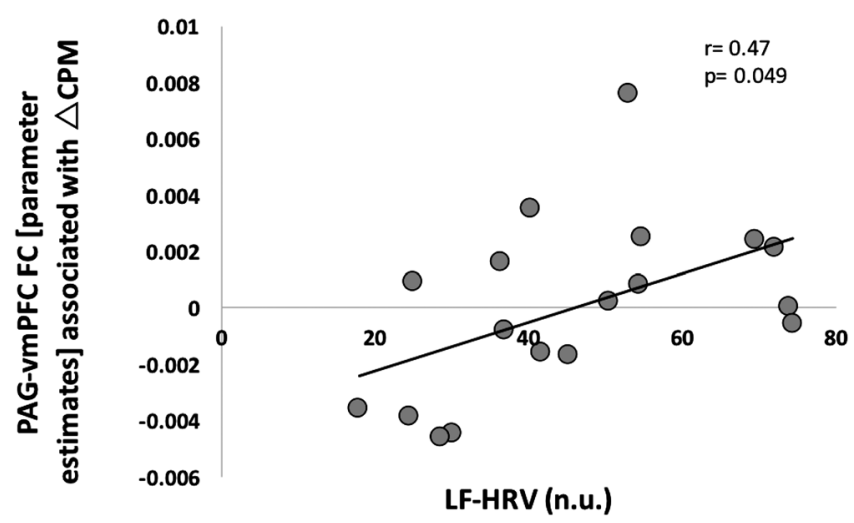

Figure 6. Correlation between LF-HRVnu and parameter estimates of CPM associated FC between PAG and vmPFC The association was investigated in $n=18$ participants, by means of Pearson's correlation coefficient.

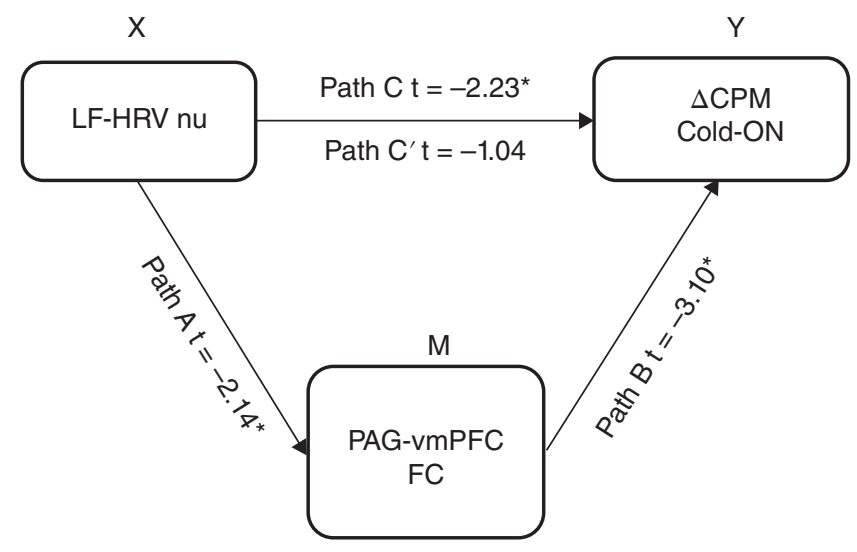

Figure 7. PAG-vmPFC functional connectivity mediates the association between LF-HRVnu and $\triangle$ CPM

Mediation analysis (performed in 18 participants) indicated that the association between autonomic reactivity during cold pain (LF-HRVnu) and the efficiency of the descending inhibitory pain mechanisms ( $\triangle C P M$ ) was strongly mediated by PAG-vmPFC functional connectivity (FC). The $t$ value for each path is presented. $* P<0.05$. also potential effects of combined ANS reactions elicited during simultaneous presentations of these stimuli and their relationship to the CPM response.

Our next aim was to investigate relationships between $\mathrm{CPM}$ response, brain FC and brain structure, and to investigate whether the $\mathrm{HRV}-\mathrm{CPM}$ relationship is mediated by a common neural network. For the FC analyses, we considered the PAG as our seed region. The PAG has a known columnar organisation with functionally discrete subfields (Bandler \& Shipley, 1994). While others have attempted to parcellate the PAG into its ventrolateral and dorsal components in their FC analyses (Segerdahl et al. 2018), this was not practical at our acquisition resolution. Here, we favoured increasing our signal to noise ratio at the cost of slightly lower spatial resolution. We suggest that individuals planning similar experiments might consider higher magnetic field strengths, (for example, 7 Tesla) and/or limited field of view acquisitions with bespoke receiver coils, to facilitate more focused investigations of PAG subfields, although note that additional challenges are likely in their implementation (Sclocco et al. 2018). The PAG is considered a core region involved in descending pain modulation that projects to the RVM, the output pathway for pain control to the dorsal horn of the spinal cord (Mtui et al. 2021). The PAG also receives cortical projections, originating principally from the mPFC (Neafsey et al. 1986; An et al. 1998); stimulation of these cortical areas can induce analgesia (Benarroch, 2012). We observed that participants who reported a reduction in pressure pain in the presence of a tonic painful conditioning stimulus, also had increased FC strength between the PAG and both the vmPFC and RVM. This result, although obtained with a more liberal cluster-forming threshold, accords with our hypotheses. Nevertheless, other fMRI studies in humans investigating brain activity underpinning CPM have provided contrasting results. In a study by Piché and colleagues (2009), a sustained increase in orbito-frontal cortex activity during the application of a cold contralateral conditioning stimulus was associated with a decrease in subjective response to evoked painful electrocutaneous test stimuli. Similarly, Bogdanov and colleagues described that individual differences in CPM (i.e. reduction in painful laser heat ratings) related to levels of PFC activation in response to the early part of the cold conditioning stimulus (Bogdanov et al. 2015). Youssef and colleagues, on the other hand, suggested that engagement of the prefrontal and cingulate areas interferes with the generation of CPM (Youssef et al. 2016). In contrast to the studies of Piche and Bogdanov, the conditioning stimulus adopted in this later study was hypertonic saline-induced muscle pain, suggesting that the discordance in results between studies is likely to relate to the specific pain modalities and/or stimulation methodology adopted in the CPM investigation 
(Kennedy et al. 2016; Kemp et al. 2019). In a resting-state fMRI study by Harper and colleagues, higher FC between the PAG, left insula and perigenual ACC was associated with more efficient inhibitory CPM responses in both healthy participants and patients with fibromyalgia, whereas PAG connectivity with the dorsal pons was associated with greater CPM inhibition only in the control group. Greater PAG connectivity to the caudal pons/rostral medulla was associated with descending pain inhibition in healthy participants, but with pain facilitation in fibromyalgia patients (Harper et al. 2018). Here, we provide new knowledge to the field by showing that the same brain structures are associated with both the cardiovascular responses to the conditioning stimulus and the efficiency of descending pain control. Our data indicate that the strength of FC between these structures is a mediator of the ANS-CPM association. Dysregulation of the three-way interaction between brain FC, efficiency of descending pain control mechanisms and autonomic reactivity might explain various symptoms of chronic pain conditions such as low HRV and impaired descending pain control compared to healthy controls (Lewis et al. 2012; Tracy et al. 2016). Future studies are required to investigate the existence and dysregulation of such a mechanism in chronic pain conditions.

Brain structure was also associated with the CPM effect. Specifically, individuals with larger vmPFC volumes exhibited an inhibitory CPM effect. Previous reports have described associations between GM volume reductions in the orbito-frontal cortex and the vmPFC (together with other areas such as the thalamus, insula, posterior cingulate cortex) and increased sensitivity to rectal pain stimuli in healthy volunteer participants (Elsenbruch et al. 2014). Reduction in vmPFC GM is found in patients with chronic complex regional pain syndrome (Geha et al. 2008), fibromyalgia (Kuchinad et al. 2007) and chronic back pain (Fritz et al. 2016). Similarly, patients with functional dyspepsia have decreased cortical thickness in prefrontal and dorso-lateral areas (Liu et al. 2018). A negative correlation was described between pain and dlPFC volumes in patients with chronic complex regional pain syndrome (Barad et al. 2014), whereas pain thresholds were correlated positively with dIPFC volumes in patients with chronic myofascial pain (Niddam et al. 2017). Here, our results show that individuals with larger vmPFC volumes report more efficient descending pain inhibition, whereas individuals with smaller GM volumes in this area showed pain facilitation during the application of the conditioning stimulus. Taken together these findings engender the working hypothesis that PFC volume reductions in multiple chronic pain conditions may be associated with impairments in descending pain control. Our data suggest that individuals with smaller vmPFC volumes exhibited pain facilitation (i.e. an increase in pain with the contralateral presentation of the conditioning stimulus), whereas those with larger vmPFC volumes exhibited a reduction in pain (indicating efficient descending pain modulating mechanisms). We speculate that, in chronic pain patients, a reduction in vmPFC volume might be involved in the phenotypic switch between descending inhibition and pathological pain facilitation (Potvin \& Marchand, 2016).

We acknowledge some methodological limitations in our study. For practical reasons, our heart rate data were acquired with a photoplethysmograph (PPG) rather than using electrocardiographic (ECG) recordings, the gold standard approach for deriving HRV measures. Some studies have shown that under certain conditions, PPG provides a good surrogate of ECG-derived HRV (Gil et al. 2010; Schäfer \& Vagedes, 2013). We encourage future studies to replicate our findings with measures obtained from ECG. We also acknowledge that the types of painful stimulation adopted in this study, that is, pressure-pain delivered to the thumb nail bed, and cold-pain delivered via an aluminum probe, should be considered in further experiments. Both stimulation modalities are non-specific, and activate nociceptors both in the surface of the skin and in the deep tissue. Studies within our team are already in progress to further explore the involvement of different types of nociceptors in the interaction between ANS and CPM.

In conclusion, this study combined rs-fMRI, recording of the ANS responses to pain and assessments of descending pain control, providing mechanistic insights into the association between the ANS and pain in the brain. Our data adds to the large body of research focusing on the link between the individual differences in autonomic balance and physical and emotional well-being, underpinned by the PFC (Thayer et al. 2009). Associations identified between CPM response, resting-state connectivity networks involved in descending pain control and flexibility in HRV suggest that individuals with stronger engagement of the ANS in response to pain are also more efficient in triggering descending pain inhibition, which may lead to more expedient recovery of homeostasis. Some of our results should be considered exploratory, given their moderate sample size and (in a subset of our imaging findings) cluster forming thresholds. We encourage their replication in a novel, independent sample. Future studies should aim to shed light on potential dysregulation of these mechanisms in patients with chronic painful conditions, which in turn may signpost future therapeutic interventions for these patients. Therapies focused on stimulation of the ANS (such as HRV biofeedback, or baroreceptor stimulation) might help to regain appropriate levels of control over not only the ANS, but also mechanisms of endogenous pain control. 


\section{References}

An X, Bandler R, Öngür D \& Price J (1998). Prefrontal cortical projections to longitudinal columns in the midbrain periaqueductal gray in macaque monkeys. J Comp Neurol 401, 455-479.

Appelhans BM \& Luecken LJ (2008). Heart rate variability and pain: associations of two interrelated homeostatic processes. Biol Psychol 77, 174-182.

Avants BB, Tustison NJ, Song G, Cook PA, Klein A \& Gee JC (2011). A reproducible evaluation of ANTs similarity metric performance in brain image registration. Neuroimage 54, 2033-2044.

Bandler R \& Shipley MT (1994). Columnar organization in the midbrain periaqueductal gray: modules for emotional expression? Trends Neurosci 17, 379-389.

Barad MJ, Ueno T, Younger J, Chatterjee N \& Mackey S (2014). Complex regional pain syndrome is associated with structural abnormalities in pain-related regions of the human brain. J Pain 15, 197-203.

Beissner F, Meissner K, Bär K-J \& Napadow V (2013). The autonomic brain: an activation likelihood estimation meta-analysis for central processing of autonomic function. J Neurosci 33, 10503-10511.

Benarroch E (1993). The central autonomic network: functional organization, dysfunction, and perspective. In Mayo Clin Proc, Elsevier. 68, 988-1001.

Benarroch E (2006). Pain-autonomic interactions. Neurol Sci 27, s130-s133.

Benarroch E (2012). Central autonomic control. In Primer on the Autonomic Nervous System, 3rd edn, pp. 9-12. Elsevier.

Bogdanov VB, Viganò A, Noirhomme Q, Bogdanova OV, Guy N, Laureys S, Renshaw PF, Dallel R, Phillips C \& Schoenen $J$ (2015). Cerebral responses and role of the prefrontal cortex in conditioned pain modulation: an fMRI study in healthy subjects. Behav Brain Res 281, 187-198.

Bouhassira D, Villanueva L, Bing Z \& le Bars D (1992). Involvement of the subnucleus reticularis dorsalis in diffuse noxious inhibitory controls in the rat. Brain Res 595, 353-357.

Bruehl S \& Chung OY (2004). Interactions between the cardiovascular and pain regulatory systems: an updated review of mechanisms and possible alterations in chronic pain. Neuroscience \& Biobehavioral Reviews 28, 395-414.

Bruehl S, Chung OY, Ward P, Johnson B \& McCubbin JA (2002). The relationship between resting blood pressure and acute pain sensitivity in healthy normotensives and chronic back pain sufferers: the effects of opioid blockade. Pain 100, 191-201.

Burr RL (2007). Interpretation of normalized spectral heart rate variability indices in sleep research: a critical review. Sleep 30, 913-919.

Chalaye P, Lafrenaye S, Goffaux P \& Marchand S (2014). The role of cardiovascular activity in fibromyalgia and conditioned pain modulation. Pain 155, 1064-1069.

Coulombe M-A, Lawrence KS, Moulin DE, Morley-Forster P, Shokouhi M, Nielson WR \& Davis KD (2017). Lower functional connectivity of the periaqueductal gray is related to negative affect and clinical manifestations of fibromyalgia. Frontiers in Neuroanatomy 11, 47.
Cox RW (1996). AFNI: software for analysis and visualization of functional magnetic resonance neuroimages. Comput Biomed Res 29, 162-173.

Dayan L, Hochberg U, Nahman-Averbuch H, Brill S, Ablin JN \& Jacob G (2018). Increased sympathetic outflow induces adaptation to acute experimental pain. Pain Pract 18, 322-330.

Dipasquale O, Sethi A, Lagana MM, Baglio F, Baselli G, Kundu P, Harrison NA \& Cercignani M (2017). Comparing resting state fMRI de-noising approaches using multi- and single-echo acquisitions. PLoS One 12, e0173289.

Edwards L, McIntyre D, Carroll D, Ring C, France CR \& Martin U (2003). Effects of artificial and natural baroreceptor stimulation on nociceptive responding and pain. Psychophysiology 40, 762-769.

Edwards RR, Ness TJ, Weigent DA \& Fillingim RB (2003). Individual differences in diffuse noxious inhibitory controls (DNIC): association with clinical variables. Pain 106, 427-437.

Eickhoff SB, Stephan KE, Mohlberg H, Grefkes C, Fink GR, Amunts K \& Zilles K (2005). A new SPM toolbox for combining probabilistic cytoarchitectonic maps and functional imaging data. Neuroimage 25, 13251335.

Elsenbruch S, Schmid J, Kullmann JS, Kattoor J, Theysohn N, Forsting M \& Kotsis V (2014). Visceral sensitivity correlates with decreased regional gray matter volume in healthy volunteers: a voxel-based morphometry study. Pain 155, 244-249.

France CR \& Katz J (1999). Postsurgical pain is attenuated in men with elevated presurgical systolic blood pressure. Pain Research and Management 4(2), 100-103.

Fritz H-C, McAuley JH, Wittfeld K, Hegenscheid K, Schmidt CO, Langner S \& Lotze M (2016). Chronic back pain is associated with decreased prefrontal and anterior insular gray matter: results from a population-based cohort study. $J$ Pain 17, 111-118.

Geha PY, Baliki MN, Harden RN, Bauer WR, Parrish TB \& Apkarian AV (2008). The brain in chronic CRPS pain: abnormal gray-white matter interactions in emotional and autonomic regions. Neuron 60, 570-581.

Ghione S (1996). Hypertension-associated hypalgesia: Evidence in experimental animals and humans, pathophysiological mechanisms, and potential clinical consequences. Hypertension 28, 494-504.

Gianaros PJ, Onyewuenyi IC, Sheu LK, Christie IC \& Critchley HD (2012). Brain systems for baroreflex suppression during stress in humans. Hum Brain Mapp 33, 1700-1716.

Gil E, Orini M, Bailon R, Vergara JM, Mainardi L \& Laguna $P$ (2010). Photoplethysmography pulse rate variability as a surrogate measurement of heart rate variability during non-stationary conditions. Physiol Meas 31, 1271.

Goldstein DS, Bentho O, Park MY \& Sharabi Y (2011). Low-frequency power of heart rate variability is not a measure of cardiac sympathetic tone but may be a measure of modulation of cardiac autonomic outflows by baroreflexes. Exp Physiol 96, 1255-1261. 
Granot M, Weissman-Fogel I, Crispel Y, Pud D, Granovsky Y, Sprecher E \& Yarnitsky D (2008). Determinants of endogenous analgesia magnitude in a diffuse noxious inhibitory control (DNIC) paradigm: do conditioning stimulus painfulness, gender and personality variables matter? Pain 136, 142-149.

Hagen K, Zwart J-A, Holmen J, Svebak S, Bovim G \& Stovner LJ (2005). Does hypertension protect against chronic musculoskeletal complaints? The Nord-Trøndelag Health Study. Arch Intern Med 165, 916-922.

Harper DE, Ichesco E, Schrepf A, Hampson JP, Clauw DJ, Schmidt-Wilcke T, Harris RE \& Harte SE (2018). Resting functional connectivity of the periaqueductal gray is associated with normal inhibition and pathological facilitation in conditioned pain modulation. J Pain 19, 635. e631-635. e615.

Harris J (1996). Descending antinociceptive mechanisms in the brainstem: their role in the animal's defensive system. $J$ Physiol Paris 90, 15-25.

Hayes AF (2012). PROCESS: A versatile computational tool for observed variable mediation, moderation, and conditional process modeling. In: University of Kansas, KS. https: //iwi.uibk.ac.at/Team/Innsbruck_Mediation_Moderation/ Hayesprocess.pdf

Hohenschurz-Schmidt DJ, Calcagnini G, Dipasquale O, Jackson JB, Medina S, O’Daly O, O’Muircheartaigh J, de Lara Rubio A, Williams SCR, McMahon SB, Makovac E \& McMahon SB (2020). Linking pain sensation to the autonomic nervous system: the role of the anterior cingulate and periaqueductal gray resting-state networks. Front Neurosci 14, 147.

Jiang C, Yi L, Su S, Shi C, Long X, Xie G \& Zhang L (2016). Diurnal variations in neural activity of healthy human brain decoded with resting-state blood oxygen level dependent fMRI. Front Hum Neurosci 10, 634.

Kemp HI, Kennedy DL, Wu C, Ridout DA \& Rice ASC (2019). Determining real change in conditioned pain modulation: a repeated measures study in healthy volunteers. J Pain 21(5-6), 708-721.

Kennedy DL, Kemp HI, Ridout D, Yarnitsky D \& Rice AS (2016). Reliability of conditioned pain modulation: a systematic review. Pain 157, 2410.

Koenig J, Jarczok MN, Ellis RJ, Hillecke T \& Thayer JF (2014). Heart rate variability and experimentally induced pain in healthy adults: a systematic review. Eur J Pain 18, 301314.

Kosek E, Rosen A, Carville S, Choy E, Gracely RH, Marcus H, Petzke F, Ingvar M \& Jensen KB (2017). Lower placebo responses after long-term exposure to fibromyalgia pain. $J$ Pain 18, 835-843.

Kuchinad A, Schweinhardt P, Seminowicz DA, Wood PB, Chizh BA \& Bushnell MC (2007). Accelerated brain gray matter loss in fibromyalgia patients: premature aging of the brain? J Neurosci 27, 4004-4007.

Kundu P, Benson BE, Baldwin KL, Rosen D, Luh WM, Bandettini PA, Pine DS \& Ernst M (2015). Robust resting state fMRI processing for studies on typical brain development based on multi-echo EPI acquisition. Brain Imaging Behav 9, 56-73.
Kundu P, Brenowitz ND, Voon V, Worbe Y, Vertes PE, Inati SJ, Saad ZS, Bandettini PA \& Bullmore ET (2013). Integrated strategy for improving functional connectivity mapping using multiecho fMRI. Proc Natl Acad Sci U S A 110, 16187-16192.

Kyle BN \& McNeil DW (2014). Autonomic arousal and experimentally induced pain: a critical review of the literature. Pain Res Manag 19, 159-167.

Laborde S, Mosley E \& Thayer JF (2017). Heart rate variability and cardiac vagal tone in psychophysiological research recommendations for experiment planning, data analysis, and data reporting. Front Psychol 08, 213-231.

Lewis GN, Rice DA \& McNair PJ (2012). Conditioned pain modulation in populations with chronic pain: a systematic review and meta-analysis. J Pain 13, 936-944.

Liu P, Wang G, Zeng F, Liu Y, Fan Y, Wei Y, Qin W \& Calhoun VD (2018). Abnormal brain structure implicated in patients with functional dyspepsia. Brain Imaging Behav 12, 459-466.

Loggia ML, Juneau M \& Bushnell MC (2011). Autonomic responses to heat pain: heart rate, skin conductance, and their relation to verbal ratings and stimulus intensity. Pain 152, 592-598.

Maixner W, Fillingim R, Kincaid S, Sigurdsson A \& Harris MB (1997). Relationship between pain sensitivity and resting arterial blood pressure in patients with painful temporomandibular disorders. Psychosom Med 59, 503-511.

Makovac E, Dipasquale O, Jackson JB, Medina S, O’Daly O, O’Muircheartaigh J, de Lara Rubio A, Williams SCR, McMahon SB \& Howard MAJB (2019). Sustained perturbation in functional connectivity induced by cold pain. Eur J Pain 24, 1850-1861.

Marciszewski KK, Meylakh N, Di Pietro F, Mills EP, Macefield VG, Macey PM \& Henderson LA (2018). Changes in brainstem pain modulation circuitry function over the migraine cycle. J Neurosci 38, 10479-10488.

Mischkowski D, Palacios-Barrios EE, Banker L, Dildine TC \& Atlas LY (2018). Pain or nociception? Subjective experience mediates the effects of acute noxious heat on autonomic responses. Pain 159(4), 699-711.

Moak JP, Goldstein DS, Eldadah BA, Saleem A, Holmes C, Pechnik S \& Sharabi YJHR (2007). Supine low-frequency power of heart rate variability reflects baroreflex function, not cardiac sympathetic innervation. Heart Rhythm 4(12), 1523-1529.

Moayedi M, Salomons TV \& Atlas LY (2018). Pain neuroimaging in humans: a primer for beginners and non-imagers. J Pain 19, 961. e961-961. e921.

Moont R, Crispel Y, Lev R, Pud D \& Yarnitsky D (2011). Temporal changes in cortical activation during conditioned pain modulation (CPM), a LORETA study. Pain 152, 1469-1477.

Mtui E, Gruener G \& FitzGerald MT (2021). Clinical Neuroanatomy and Neuroscience E-Book. 8, Elsevier Health Sciences.0-432.

Nahman-Averbuch H, Yarnitsky D, Sprecher E, Granovsky Y \& Granot M (2016). Relationship between personality traits and endogenous analgesia: the role of harm avoidance. Pain Pract 16, 38-45. 
Neafsey EJ, Hurley-Gius KM \& Arvanitis D (1986). The topographical organization of neurons in the rat medial frontal, insular and olfactory cortex projecting to the solitary nucleus, olfactory bulb, periaqueductal gray and superior colliculus. Brain Res 377, 261-270.

Niddam D, Lee SH, Su YT \& Chan RC (2017). Brain structural changes in patients with chronic myofascial pain. Eur J Pain 21, 148-158.

Oldfield RC (1971). The assessment and analysis of handedness: the Edinburgh inventory. Neuropsychologia 9, 97-113.

Olsen RB, Bruehl S, Nielsen CS, Rosseland LA, Eggen AE \& Stubhaug A (2013). Gender differences in blood pressure-related hypoalgesia in a general population: the Tromsø study. J Pain 14, 699-708.

Ottaviani C, Fagioli S, Mattei E, Censi F, Edwards L, Macaluso E, Bozzali M, Critchley HD \& Calcagnini G (2018). Brain-heart pathways to blood pressure-related hypoalgesia. Psychosom Med 80, 845-852.

Peyron R, Laurent B \& Garcia-Larrea L (2000). Functional imaging of brain responses to pain. A review and meta-analysis (2000). Neurophysiologie Clinique/Clinical Neurophysiology 30, 263-288.

Piché M, Arsenault M \& Rainville P (2009). Cerebral and cerebrospinal processes underlying counterirritation analgesia. J Neurosci 29, 14236-14246.

Posse S, Wiese S, Gembris D, Mathiak K, Kessler C, Grosse-Ruyken ML, Elghahwagi B, Richards T, Dager SR \& Kiselev VG (1999). Enhancement of BOLD-contrast sensitivity by single-shot multi-echo functional MR imaging. Magn Reson Med 42, 87-97.

Potvin S \& Marchand S (2016). Pain facilitation and pain inhibition during conditioned pain modulation in fibromyalgia and in healthy controls. Pain 157, 1704-1710.

Rahman F, Pechnik S, Gross D, Sewell L \& Goldstein DS (2011). Low frequency power of heart rate variability reflects baroreflex function, not cardiac sympathetic innervation. Clin Auton Res 21, 133-141.

Reis DJ, Ruggiero DA \& Morrison SF (1989). The CI area of the rostral ventrolateral medulla oblongata: a critical brainstem region for control of resting and reflex integration of arterial pressure. Am J Hypertens 2, 363S-374S.

Reyes del Paso GA, Langewitz W, Mulder LJ, van Roon A \& Duschek S (2013). The utility of low frequency heart rate variability as an index of sympathetic cardiac tone: a review with emphasis on a reanalysis of previous studies. Psychophysiology 50, 477-487.

Roy M, Piché M, Chen J-I, Peretz I \& Rainville P (2009). Cerebral and spinal modulation of pain by emotions. Proc Natl Acad Sci U S A 106, 20900-20905.

Saccò M, Meschi M, Regolisti G, Detrenis S, Bianchi L, Bertorelli M, Pioli S, Magnano A, Spagnoli F, Giuri PG, Fiaccadori E \& Giuri PG (2013). The relationship between blood pressure and pain. J Clin Hypertens 15, 600-605.

Schäfer A \& Vagedes J (2013). How accurate is pulse rate variability as an estimate of heart rate variability?: a review on studies comparing photoplethysmographic technology with an electrocardiogram. Int J Cardiol 166, 15-29.
Sclocco R, Beissner F, Bianciardi M, Polimeni JR \& Napadow $\mathrm{V}$ (2018). Challenges and opportunities for brainstem neuroimaging with ultrahigh field MRI. Neuroimage 168, 412-426.

Segerdahl AR, Themistocleous AC, Fido D, Bennett DL \& Tracey I (2018). A brain-based pain facilitation mechanism contributes to painful diabetic polyneuropathy. Brain 141, 357-364.

Siegel JS, Mitra A, Laumann TO, Seitzman BA, Raichle M, Corbetta M \& Snyder AZ (2016). Data quality influences observed links between functional connectivity and behavior. Cereb Cortex 27(9), 4492-4502.

Sklerov M, Dayan E \& Browner NJCAR (2018). Functional neuroimaging of the central autonomic network: recent developments and clinical implications. Clin Auton Res $1-12$.

Smith SM, Jenkinson M, Woolrich MW, Beckmann CF, Behrens TE, Johansen-Berg H, Bannister PR, Luca MD, Drobnjak I, Flitney DE, Niazy RK, Saunders J, Vickers J, Zhang Y, Stefano ND, Brady JM \& Flitney DE (2004). Advances in functional and structural MR image analysis and implementation as FSL. Neuroimage 23, S208S219.

Spielberger C, Gorsuch R, Lushene R, Vagg P \& Jacobs G (1983). Manual for the State-Trait Anxiety Inventory. (Consulting Psychologists Press, Palo Alto, CA, USA.

Strian F, Lautenbacher S, Galfe G \& Hölzl R (1989). Diurnal Variations in Pain Perception and Thermal Sensitivity. Pain Bamberg. 36(1), 125-131.

Suarez-Roca H, Klinger RY, Podgoreanu MV, Ji R-R, Sigurdsson MI, Waldron N, Mathew JP \& Maixner W (2019). Contribution of baroreceptor function to pain perception and perioperative outcomes. Anesthesiol 130, 634-650.

Tarvainen MP, Niskanen J-P, Lipponen JA, Ranta-Aho PO \& Karjalainen PA (2014). Kubios HRV-heart rate variability analysis software. Comput Methods Programs Biomed 113(1), 210-220.

Thayer JF, Hansen AL, Saus-Rose E \& Johnsen BH (2009). Heart rate variability, prefrontal neural function, and cognitive performance: the neurovisceral integration perspective on self-regulation, adaptation, and health. Ann Behav Med 37, 141-153.

Thayer JF \& Lane RD (2000). A model of neurovisceral integration in emotion regulation and dysregulation. J Affect Disord 61(3), 201-216.

Thissen D, Steinberg L \& Kuang D (2002). Quick and easy implementation of the Benjamini-Hochberg procedure for controlling the false positive rate in multiple comparisons. Journal of Educational and Behavioral Statistics 27, 77-83.

Tobaldini G, Sardi NF, Guilhen VA \& Fischer L (2019). Pain inhibits pain: an ascending-descending pain modulation pathway linking mesolimbic and classical descending mechanisms. Mol Neurobiol 56, 1000-1013.

Tousignant-Laflamme Y \& Marchand S (2009). Excitatory and inhibitory pain mechanisms during the menstrual cycle in healthy women. Pain 146, 47-55. 
Tracy LM, Ioannou L, Baker KS, Gibson SJ, Georgiou-Karistianis N \& Giummarra MJ (2016). Meta-analytic evidence for decreased heart rate variability in chronic pain implicating parasympathetic nervous system dysregulation. Pain 157, 7-29.

Traxler J, Hanssen MM, Lautenbacher S, Ottawa F \& Peters ML (2019). General versus pain-specific cognitions: pain catastrophizing but not optimism influences conditioned pain modulation. Eur J Pain 23, 150-159.

Villanueva L, Bouhassira D \& Le Bars D (1996). The medullary subnucleus reticularis dorsalis (SRD) as a key link in both the transmission and modulation of pain signals. Pain 67, 231-240.

Vincent K \& Tracey I (2010). Sex hormones and pain: the evidence from functional imaging. Curr Pain Headache Rep 14(5), 396-403.

Worsley KJ \& Friston KJ (1995). Analysis of fMRI time-series revisited-again. Neuroimage 2(3), 173-181.

Yarnitsky D (2010). Conditioned pain modulation (the diffuse noxious inhibitory control-like effect): its relevance for acute and chronic pain states. Curr Opin Anaesthesiol 23, 611-615.

Yarnitsky D, Bouhassira D, Drewes A, Fillingim R, Granot M, Hansson P, Landau R, Marchand S, Matre D, Nilsen KB, Stubhaug A, Treede RD \& Nilsen K (2015). Recommendations on practice of conditioned pain modulation (CPM) testing. Eur J Pain 19, 805-806.

Yarnitsky D, Crispel Y, Eisenberg E, Granovsky Y, Ben-Nun A, Sprecher E, Best L-A \& Granot M (2008). Prediction of chronic post-operative pain: pre-operative DNIC testing identifies patients at risk. Pain 138, 22-28.

Yarnitsky D, Granot M, Nahman-Averbuch H, Khamaisi M \& Granovsky Y (2012). Conditioned pain modulation predicts duloxetine efficacy in painful diabetic neuropathy. Pain $\mathbf{1 5 3}$ 1193-1198.

Yelle MD, Oshiro Y, Kraft RA \& Coghill RC (2009). Temporal filtering of nociceptive information by dynamic activation of endogenous pain modulatory systems. J Neurosci 29, 10264-10271.

Youssef AM, Macefield VG \& Henderson LA (2016). Cortical influences on brainstem circuitry responsible for conditioned pain modulation in humans. Hum Brain Mapp 37, 2630-2644.

\section{Additional information}

\section{Data availability statement}

The data that support the findings of this study are available from the corresponding author upon reasonable request.

\section{Competing interest}

None.

\section{Author contributions}

E.M.: design of the experiment, data collection, data analysis and manuscript preparation. A.V.: data collection. D.H.S., O.D. and O.O.D.: data analysis and manuscript preparation. J.B.J.: design of the experiment, data collection, data analysis and manuscript preparation. S.M.: data collection and manuscript preparation. S.C.R.W., S.S.B. and M.A.H.: supervised the experiment planning, data collection, data analysis and manuscript preparation. All authors have approved the final version of the manuscript and agree to be accountable for all aspects of the work. All persons designated as authors qualify for authorship, and all those who qualify for authorship are listed.

\section{Funding}

This work was funded by a Medical Research Council Experimental Medicine Challenge Grant (MR/N026969/1). M.A.H., S.M. and S.W. are also supported by the NIHR Biomedical Research Centre for Mental Health at the South London and Maudsley NHS Trust. E.M. is also supported by an EFIC-Grünenthal-Grant.

\section{Acknowledgements}

The authors also wish to thank the Wellcome Trust for their ongoing support of our neuroimaging research and Simon Hill for both the development and calibration of the stimulation equipment and Giovanni Calcagnini for his input on heart rate variability analysis.

\section{Keywords}

descending pain modulation, heart rate variability, periaqueductal grey, resting state, voxel-based morphometry

\section{Supporting information}

Additional supporting information can be found online in the Supporting Information section at the end of the HTML view of the article. Supporting information files available:

\section{Peer Review History Statistical Summary Document}

